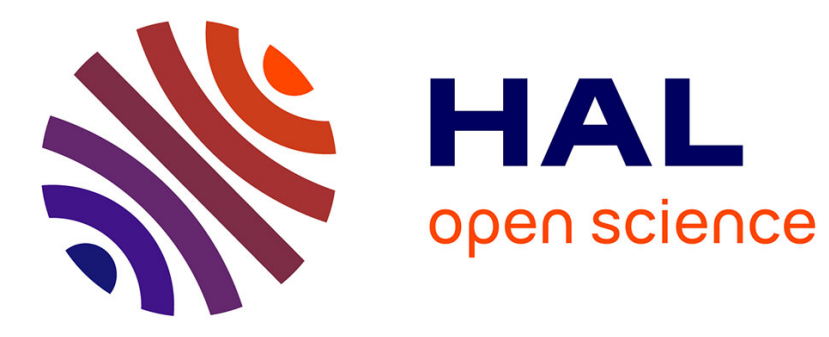

\title{
Sandstorm effect on experimental optical camera communication
}

Vicente Matus, Victor Guerra, Stanislav Zvanovec, Jose Rabadan, Rafael Perez-Jimenez

\section{- To cite this version:}

Vicente Matus, Victor Guerra, Stanislav Zvanovec, Jose Rabadan, Rafael Perez-Jimenez. Sandstorm effect on experimental optical camera communication. Applied optics, 2021, 60 (1), pp.75. 10.1364/AO.405952 . hal-03341312

\section{HAL Id: hal-03341312 \\ https://hal-amu.archives-ouvertes.fr/hal-03341312}

Submitted on 10 Sep 2021

HAL is a multi-disciplinary open access archive for the deposit and dissemination of scientific research documents, whether they are published or not. The documents may come from teaching and research institutions in France or abroad, or from public or private research centers.
L'archive ouverte pluridisciplinaire HAL, est destinée au dépôt et à la diffusion de documents scientifiques de niveau recherche, publiés ou non, émanant des établissements d'enseignement et de recherche français ou étrangers, des laboratoires publics ou privés. 


\title{
Sandstorm effect on experimental optical camera communication
}

\author{
Vicente Matus $^{1}{ }^{*}$, Victor Guerra $^{1}$, Stanislav Zvanovec ${ }^{2}$, Jose Rabadan ${ }^{1}$, And Rafael \\ PeREZ-JIMENEZ ${ }^{1}$ \\ ${ }^{1}$ IDeTIC, Universidad de Las Palmas de Gran Canaria, Spain \\ ${ }^{2}$ Czech Technical University in Prague, Czech Republic \\ *Corresponding author: vmatus@idetic.eu \\ Compiled January 11, 2021
}

Sandstorms can severely affect outdoor Optical Wireless Communications (OWC) reliability by diminishing large regions' visibility. In this work, the effect of a real sandstorm on Optical Camera Communications (OCC) links is experimentally evaluated. Two link ranges are essayed using a cost-efficient telescope-based camera setup with commercial LEDs. Using On-Off Keying modulation, a data rate of 1035 and $630 \mathrm{bps}$ with error probabilities of $9.14 \cdot 10^{-5}$ and $4.1 \cdot 10^{-3}$ for $100 \mathrm{~m}$ and $200 \mathrm{~m}$ respectively can be achieved. The Signal-to-Noise Ratio of the links was optimized by tuning the analog amplifier's gain of the camera, increasing it by up to $9 \mathrm{~dB}$. It is shown that the scattering due to the sandstorm can be even beneficial for increasing the data rate in OCC (contrary to classical photodetector-based OWC links) thanks to an increment of $33 \%$ on the Region of Interest dimensions compared to the expected clear-air link. (๑) 2021 Optical Society of America

http://dx.doi.org/10.1364/ao.XX.XXXXXX

\section{INTRODUCTION}

Optical Camera Communication (OCC) is an essential sub-field of Visible Light Communication (VLC). It is based on the use of image sensors as receivers of the optical data signal. The ubiquity of camera devices in consumer electronics (e.g. smartphones, tablets, and laptops) is expected to bring this technology to the mass market earlier than other VLC systems that need a photodiode (PD) or multiple PDs as receivers, which are not available in commercial off-the-shelf devices. OCC is currently part of the Institute of Electrical and Electronics Engineers (IEEE) 802.15.7r1 standard [1], defining several transmission modes taking into account the particular characteristics of the image sensor, such as the Rolling Shutter (RS) and Global Shutter (GS) scanning methods. RS exploits the Complementary MetalOxide-Semiconductor (CMOS) sensor's progressive scanning of the image, which is done on a row-by-row basis, meaning that each row's exposure starts after the previous one's by a delay known as the row-shift time, and it enables communications up to several kbps [2]. GS is used in Charge-Coupled Device (CCD) sensors, which expose the whole frame simultaneously. Thus, the frame rate limits GS' throughput to tens or hundreds of bps. RS techniques provide higher data rates compared to GS since each image frame can contain several rows capturing different states of the transmitter. RS' achievable data rate is then highly dependent on the optical source's projection over the image sensor's scanning axis because this size determines the number of detectable bits in the image frame [3]. The camera's optical system can augment the projection by, for example, defocusing and magnifying the image [4].

This low-speed technology has been proposed as suitable for many indoor applications such as visible light positioning, wireless monitoring of patients' sensors in hospitals, and peerto-peer data sharing, among others [2]. Furthermore, OCC is capable of operating in outdoor environments using the appropriate settings of the camera $[5,6]$. Its potential use on vehicular communications has been recurrently reported in the literature $[2,7]$. Moreover, cameras' inherent spatial multiplexing capability has been exploited to define sensor monitoring applications in Smart Cities [8]. The vehicular application of VLC has been studied in [9], where different scenarios of atmospheric conditions have been simulated. The PD-based receiver link achieves a maximum distance of about $40 \mathrm{~m}$ in clear weather conditions for a vehicle-to-vehicle communication scenario if a $10^{-4} \mathrm{Bit}$ Error Rate (BER) is required. In harsh conditions, the maximum achievable distance drops to around $35 \mathrm{~m}$, which is shown to increase to about $90 \mathrm{~m}$ if optical gain techniques such as increasing the focal aperture.

Free Space Optics (FSO) employing photodiodes and lasers are one of the most advanced Optical Wireless Communications (OWC) for outdoor applications, with data rates around $10 \mathrm{Gbps}$ and proven reliability even using mobile nodes in link spans from a few hundred meters to tens of $\mathrm{km}[10,11]$. Despite its 
very low data rates, OCC can still be competitive due to its cost-effectiveness. This technology can use inexpensive and already-deployed devices such as LED lighting and ubiquitous digital cameras, as in most of the aforementioned applications.

Atmospheric conditions that may affect OWC and FSO performance are uncontrollable, but they must be considered when designing outdoor optical systems. The most studied phenomena are fog, mist, turbulence, aerosols, and precipitations (rain and snow). The joint impact of atmospheric turbulence and particle-related scattering in FSO communications at infrared wavelengths has been extensively measured by Libich et al. in [12], emulating smog particles and turbulence conditions in a laboratory chamber, using sand and ashes at different densities, and airflow at different velocities. Real outdoor scenarios have been experimentally studied for OCC in [4, 8]. Chavez-Burbano et al. employed a GS camera under clean atmospheric conditions, and a $328 \mathrm{~m}$-long link achieved a data rate of 15 bps with an error of approximately $4 \%$. Eso et al. established a $400 \mathrm{~m}$-long link with 450 bps data rate and null error using an RS in clear weather. The camera lens was used to defocus the image and obtain a bigger projection of the object in the image sensor plane.

The experimental work on overcoming the attenuation of the VLC link caused by atmospheric conditions has been implemented using optical gain by Kim et al. [13], despite that the field-of-view of the photodetector-based receiver is reduced. A different strategy has been developed for OCC in [5, 14], where the use of the camera's analog gain was evaluated in the laboratory under fog conditions. The results showed that this parameter's optimization could significantly improve the signal quality in scenarios subject to high fog attenuation and particles scattering.

In high mobility scenarios such as OCC's vehicular application, the Region of Interest (ROI) detection is an essential feature for reliable links. The ROI consists of the image pixels that contain data, i.e. where the light rays from the transmitter impinge at the camera's image sensor, which can only be known beforehand in scenarios where the node positions are fixed. The use of Neural Networks (NN) to detect the ROI by finding well-known signals contained in an image frame has allowed researchers to implement physical layer modes from IEEE 802.15.7r1 (TG7r1) [15]. The system architecture proposed includes a two-stage NN to decode bits in pictures taken with an RS camera, although it has not been tested in real-world conditions. The effect of a sandstorm on VLC links was simulated by Ebrahim et al. in [16]. The authors studied the use case of vehicular-to-road communication under different particle types of sandstorms, which differed in size and density. High-density clay particles of $2 \mu \mathrm{m}$ diameter lowered the maximum achievable span to $90 \mathrm{~m}$ in the simulation. On the other hand, Ghassemlooy et al. replicated sandstorm conditions in a laboratory environment for evaluating the effect of this phenomenon on FSO links [17]. Contrary to VLC or FSO, this phenomenon has never been theoretically addressed nor experimentally evaluated for OCC systems.

This paper provides the first experimental investigation (up to the authors' knowledge) that analyzes the impact of a sandstorm event on an OCC link at long ranges. The main contribution is to measure and prove the effects of the phenomenon in the optical wireless channel for the OCC link and define the essential blocks for the camera's digital signal processor that ensure the detection of data containing pixels in the ROI of the image frames. This assessment is fundamental for OCC applications in Smart Cities to be developed and deployed.

This paper is organized as follows. Section 2 explains the channel model for the RS optical camera communication, considering the presence of sandstorm conditions. In Section 3, we characterize the experimental scenario and derive the data analysis flow. Section 4 shows the outcome of the deployed OCC experiment in real sandstorm conditions. Finally, conclusions are summarized in Section 5.

\section{CHANNEL MODEL}

In this section, the optical wireless channel is modeled for an outdoor setting regarding the signal's attenuation due to sandstorm particles. The signal quality metrics are derived from the configuration of the transmitter $\left(T_{x}\right)$ and receiver $\left(R_{x}\right)$ units, taking into consideration the ROI dimensions of width and height.

The light pulses of varying wavelength $\lambda$ generated by the transmitter are propagated through the medium and suffer from attenuation $a(\lambda)$, as modeled by Beer's law [18], and from scattering $b(\lambda)$ caused by the particles $[19,20]$. The extinction coefficient $K_{\text {ext }}(\lambda)$ of an optical wireless channel is then modeled as:

$$
K_{\text {ext }}(\lambda)=a(\lambda)+b(\lambda) .
$$

Note that the multiple particles along the path can cause nonline-of-sight rays of light to eventually impinge on the receiver's surface, which can be advantageous for FSO links, as reported by Kedar and Arnon in [21], and potentially for OCC. In the case of OCC in multiple-scattering scenarios, the overall received signal may be improved by expanding the apparent dimensions of bright objects. The power signal at the receiver $P_{R x}(t)$ can be modeled as:

$$
P_{R x}(t)=P_{T x}(t) \cdot e^{-K_{e x t}(\lambda) \cdot d} \cdot R(\theta, \phi) \cdot \frac{A_{l e n s} \cos \Psi}{d^{2}},
$$

where the transmitter is modeled as a source of light with a predetermined radiation pattern $R(\theta, \phi)$ and power $P_{T x}(t)$, located at a distance $d$ of the receiver. The variables $\theta$, and $\phi$ are the emission angles, $\Psi$ is the incident angle, $A_{l e n s}$ is the area of the receiver's external lens. The extinction coefficient at $550 \mathrm{~nm}$ can be related to Visibility (V)[14] as:

$$
V=\frac{3.94}{K_{\text {ext }}(\lambda=550 \mathrm{~nm})} .
$$

In order to determine the signal quality, the Signal-to-Noise Ratio (SNR) was computed using the ROI detected within the image frame. The RS camera captures (in line of sight) an On-Off keying (OOK) modulated transmitted signal. The approximation of the SNR has been derived (note the 1/2 factor due to OOK) as:

$$
S N R=\frac{1}{2} \frac{M_{2}\left[X_{R O I}-\mu_{b}\right]}{V\left[X_{R O I}\right]},
$$

where $X_{R O I}$ are the values of pixels that fall within the ROI, $\mu_{b}$ is the mean of the background offset, and $M_{2}[\cdot]$, and $V[\cdot]$ denote the second-order momentum and variance, respectively.

According to the expected theoretical BER [22], the OOK system's error rate is then given by:

$$
B E R=\frac{1}{2} \operatorname{erfc}\left(\sqrt{\frac{S N R}{2}}\right),
$$

where $\operatorname{erfc}(\cdot)$ is the complementary error function.

If the architecture of the camera pixels is taken into account, the main noise sources are the thermal noise $\left(\sigma_{t h}^{2}\right)$, the shot noise 
$\left(\sigma_{s h}^{2}\right)$, and the quantization noise $\left(\sigma_{a d c}^{2}\right)$ induced by the AnalogDigital Converter (ADC). Considering that the camera has an amplifier with analog gain $G_{V}$, the SNR at any pixel is expressed as:

$$
S N R_{p x} \approx \frac{G_{V} \cdot i_{p d}^{2}}{G_{V}\left(\sigma_{t h}^{2}+\sigma_{s h}^{2}\right)+\sigma_{a d c}^{2}},
$$

where $i_{p d}$ is the current generated by the photodiode of the pixel when it is illuminated by the optical communication signal without external interfering sources.

It can be seen that by increasing the analog gain of the camera, the relative contribution of $\sigma_{a d c}^{2}$ can be reduced, and the SNR can reach an asymptotic value of $i_{p d}^{2} /\left(\sigma_{t h}^{2}+\sigma_{s h}^{2}\right)$ in case the ADC is not saturated. This can be exploited for improving the SNR in highly-attenuated scenarios when the received signal is weak and the quantization noise needs to be lowered. Furthermore, the maximum theoretical SNR improvement is given by

$$
\Delta S N R \approx 1+\frac{\sigma_{a d c}^{2}}{\sigma_{t h}^{2}+\sigma_{s h}^{2}} .
$$

Using the model and metrics stated in this section, the experimental validation was carried out in the sandstorm scenario following the methodology detailed in Section 3.

\section{METHODOLOGY}

In this section, the scenario of experimentation in the real conditions of a sandstorm is described. The OCC system used for the assessment of signal quality is characterized as well. Other well-known mathematical tools used in the data processing flow are also introduced.

A sandstorm severely hit the Canary Islands archipelago from the Sahara desert for several days at the end of February 2020. The visibility dropped to tens of meters in some places, according to the United States' National Aeronautics and Space Administration (NASA) Earth Observatory [23]. The airport of Gran Canaria reported a visibility minimum of around $0.4 \mathrm{~km}$, affecting more than 300 flights in the island, and more than 800 in the whole archipelago. This event, known as "Calima" by the islanders, was said to be the worst in four decades.

The experimental setup shown in Fig. 1 and detailed in Table 1 was deployed outdoors near the Institute for Technological Development and Innovation in Communications (IDeTIC) from the University of Las Palmas de Gran Canaria (ULPGC), whose location is shown in Fig. 2. The system consisted of a Light-Emitting Diode (LED) transmitter and a telescope and CMOS camera receiver. The emitter was based on a rectangular lamp with Red, Green, and Blue (RGB) LED strips with a white diffuser on top and controlled by an OOK signal generator, programmed to send a fixed and repetitive pattern or beacon of pure G-R-B pulses followed by a black pulse during the experiments easing both pre-processing and data analysis tasks since a well-known signal is expected. On the other hand, a CMOS image sensor was used as a receiver. A Galilean telescope with a focal length of $700 \mathrm{~mm}$ was coupled to the camera in order to reach the target distances of the experiment of $d_{1}=100 \mathrm{~m}$ and $d_{2}=200 \mathrm{~m}$.

Regarding the channel conditions at the time of experimentation, and according to the Barcelona Dust Forecast Center from the Spanish State Meteorological Agency (AEMET), the key parameters of the sandstorm, as modelled as an aerosol particle of median size of $2.524 \mu \mathrm{m}$ and 2.0 geometric standard deviation [24], are the following. The dust Aerosol Optical Depth at $\lambda=550 \mathrm{~nm}\left(\mathrm{AOD}_{550}\right)$ was between 3.2 and 6.4. This parameter increases with particles' concentration in the air, and clear air values are usually below 0.1 . The dust surface concentration was between 500 and $2000 \mu \mathrm{g} / \mathrm{m}^{3}$, with a dry deposition from 100 to $400 \mathrm{mg} / \mathrm{m}^{2}$.

The experiment's main target was to observe the sandstorm's impact on an outdoor OCC link's performance. As it occurs in other environments where the medium presents significant particle concentrations, sandstorm-impaired OCC links will suffer from multiple scattering. Hence, it was expected that the pixel SNR diminished with distance and that the Point Spread Function (PSF) of the emitter-receiver pair widened with distance, resulting in a greater-than-expected Region-Of-Interest (ROI).

\begin{tabular}{|c|c|c|}
\hline Module & Parameter & Value \\
\hline \multirow[t]{5}{*}{$T_{x}$} & Emitter dimensions & $4.2 \mathrm{~cm} \times 45 \mathrm{~cm}$ \\
\hline & Average source radiance & $10 \mathrm{~W} / \mathrm{m}^{2}$ \\
\hline & Transmitting devices & RGB LED strips \\
\hline & & $(108 \times 5050$ SMD chips $)$ \\
\hline & LED dominant wavelengths [nm] & $630(\mathrm{R}), 530(\mathrm{G}), 475(\mathrm{~B})$ \\
\hline \multirow[t]{4}{*}{ Channel } & Distance $(\mathrm{d})$ & $100 \mathrm{~m}, 200 \mathrm{~m}$ \\
\hline & Aerosol Optical Depth (AOD 550$)$ & 3.2 to $6.4(\lambda=550 \mathrm{~nm})$ \\
\hline & Dust surface concentration $\left[\mu \mathrm{g} / \mathrm{m}^{3}\right]$ & 500 to 2000 \\
\hline & Dust dry deposition $\left[\mathrm{mg} / \mathrm{m}^{2}\right]$ & 100 to 400 \\
\hline \multirow[t]{7}{*}{$R_{x}$} & Telescope focal length $(f)$ & $700 \mathrm{~mm}$ \\
\hline & Telescope focal aperture (D) & $f / 11.6$ \\
\hline & Image sensor model & Sony IMX219 [25] \\
\hline & Image sensor resolution & $2592 \times 1952 \mathrm{px}$ \\
\hline & RS row-shift time $\left(t_{r s}\right)$ & $18.904 \mu \mathrm{s}$ \\
\hline & Camera Gain $\left(G_{V}\right)$ & $0, \ldots, 20.6 \mathrm{~dB}$ \\
\hline & Exposure time $\left(t_{\text {exp }}\right)$ & $85 \mu \mathrm{s}$ \\
\hline
\end{tabular}

Table 1. Parameters of the experiment modules

The experimental evaluation was divided into three phases: data acquisition, pre-processing, and data analysis. Data acquisition was automated using a script on the receiver side, obtaining 50 images per analog gain $G_{V}$. Due to the emitter's shape, the long link range, and the weather conditions, the captured images showed the emitter in variable positions and rotations (caused by the wind perturbations). In order to solve this and provide only the dependence of the sandstorm scattering, a semi-supervised pre-processing of the images was carried out. The emitter rotations were estimated using image-processing software, and the rows were shifted according to the estimated angular deviation of up to $4^{\circ}$. Figure 3 illustrates the pre-processing stage with the removal of angular misalignment due to wind perturbations, which also included an arbitrary pre-ROI crop. Finally, all the image database was batch-processed (in terms of $G_{V}$ ), estimating both the average pixel SNR and the ROI's size.

In order to obtain the pixel SNR, the ROI was needed to be distinguished between the background and the transmitted signal. After empirically analyzing the shape of the image histograms, such as the plot in Fig. 4, it was observed that a Gaussian Mixture Model (GMM) could be used to separate the two stated classes (background and signal). The fitted Probability Density Function (PDF) can be described as: 


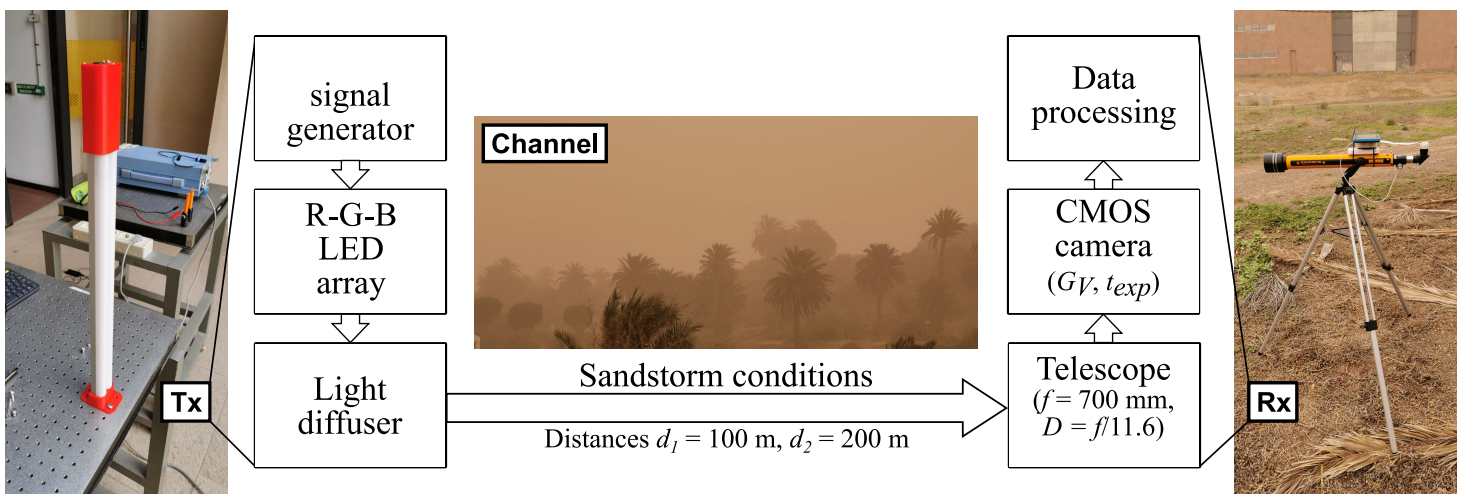

Fig. 1. Block diagram and pictures of the experimental setup of this work.

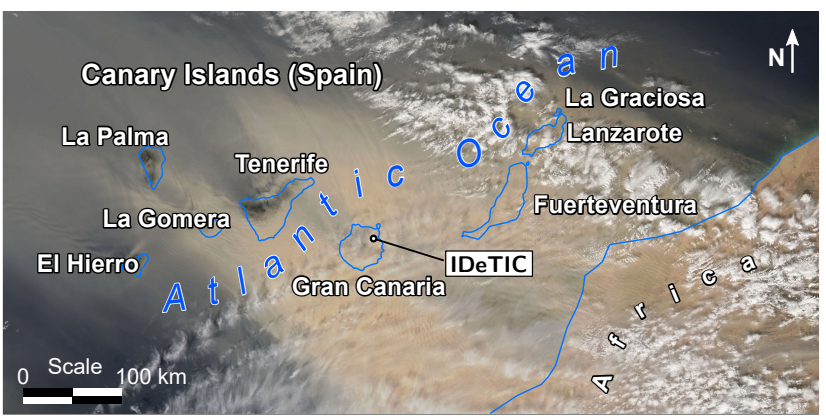

(a)

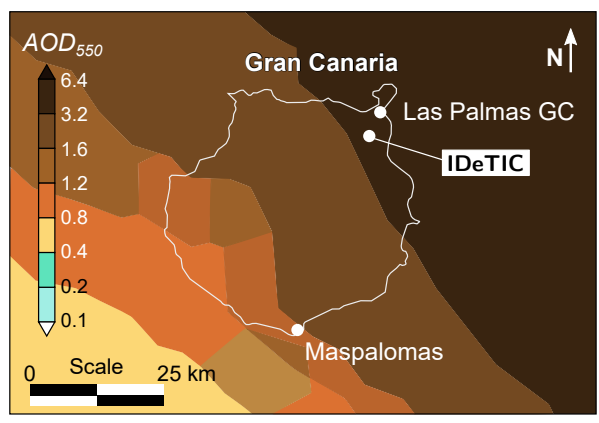

(b)

Fig. 2. Maps from the sandstorm event in the Canary Islands, February $23^{r d}, 2020$.

(a) Satellite image from NASA Moderate Resolution Imaging Spectroradiometer (MODIS) showing the dust in real colors.

(b) Map of Aerosol Optical Depth at $550 \mathrm{~nm}\left(\mathrm{AOD}_{550}\right)$ at the time of experimentation, provided by AEMET. Approximate location of the IDeTIC facilities is shown in the maps.

$$
\begin{aligned}
f_{g m m}(x)= & \frac{\alpha}{\sigma_{b} \sqrt{2 \pi}} \exp \left(-\frac{\left(x-\mu_{b}\right)^{2}}{2 \sigma_{b}^{2}}\right)+ \\
& \frac{1-\alpha}{\sigma_{s} \sqrt{2 \pi}} \exp \left(-\frac{\left(x-\mu_{s}\right)^{2}}{2 \sigma_{s}^{2}}\right)
\end{aligned}
$$

where $\mu$ is the mean and $\sigma$ is the standard deviation of the Gaussian functions. The sub-indices $b$ and $s$ label background and signal classes, respectively. The parameter $\alpha=0.9$ is an arbitrary weight of the fitting.

From the PDF description, it is straightforward to obtain the optimal decision threshold $x_{t h}$ attending to the maximum likelihood criterion, which results in a second-order equation:

$$
\begin{aligned}
0= & \left(\sigma_{b}^{2}-\sigma_{s}^{2}\right) x_{t h}^{2}+2\left(\mu_{b} \sigma_{s}^{2}-\mu_{s} \sigma_{b}^{2}\right) x_{t h}+\mu_{s}^{2} \sigma_{b}^{2} \\
& -\mu_{b}^{2} \sigma_{s}^{2}+2 \sigma_{s}^{2} \sigma_{b}^{2} \ln \left(\frac{\alpha}{1-\alpha} \frac{\sigma_{s}}{\sigma_{b}}\right)
\end{aligned}
$$

Using the calculated $x_{t h}$, all the pixels were classified into background or signal classes. The ROI was estimated as the total number of contiguous samples belonging to the signal class (on the $x$-axis) with the maximum cardinality. This implies statistical filtering that avoids possible glitches on the classification of the actual background samples. The ROI height was estimated as the number of correctly detected beacons using a template-matching correlator.

As was aforementioned, the PSF would vary by a widening factor as a function of distance. However, in a multiplescattering-free atmospheric link, the product of the ROI width $w$, and the link distance is constant. Due to the experiment's nature, Welch's T-Test was performed to prove the expected nonlinearity. The T-Test outputs a p-value $\in(0,1)$, which is closer to 0 when the null hypothesis is more likely to be incorrect. Equation 10 describes the null hypothesis (two-tailed test), in which $\mu_{w}^{(d)}$ is the average ROI width of the population at distance $d$.

$$
H_{0}: d_{1} \cdot \mu_{w}^{\left(d_{1}\right)}=d_{2} \cdot \mu_{w}^{\left(d_{2}\right)}
$$

The ROI width expansion was assessed only for the green channel $(530 \mathrm{~nm})$ without loss of generality. Particle size distributions during sandstorms [26] present modal diameters much larger than $1 \mu \mathrm{m}$, which is the upper limit for considering wavelength-dependent scattering effects (Mie or Rayleigh). Moreover, the probability of finding a particle with a diameter below this limit is much smaller than finding large particles. Hence, the ROI width would not significantly vary with respect to wavelength.

Regarding the pixel SNR, Eq. 4 showed its mathematical description. As commented, in order to have a good approximation, the background level (lamp OFF state) must be obtained. The parameter $\mu_{b}$ is reused to estimate the level. All the pixels classified into the signal class during the GMM procedure were used to estimate both signal and noise powers.

Finally, Pearson's correlation coefficient $r_{x y}$, which was used as link-quality estimator as in [14], because its value increases 


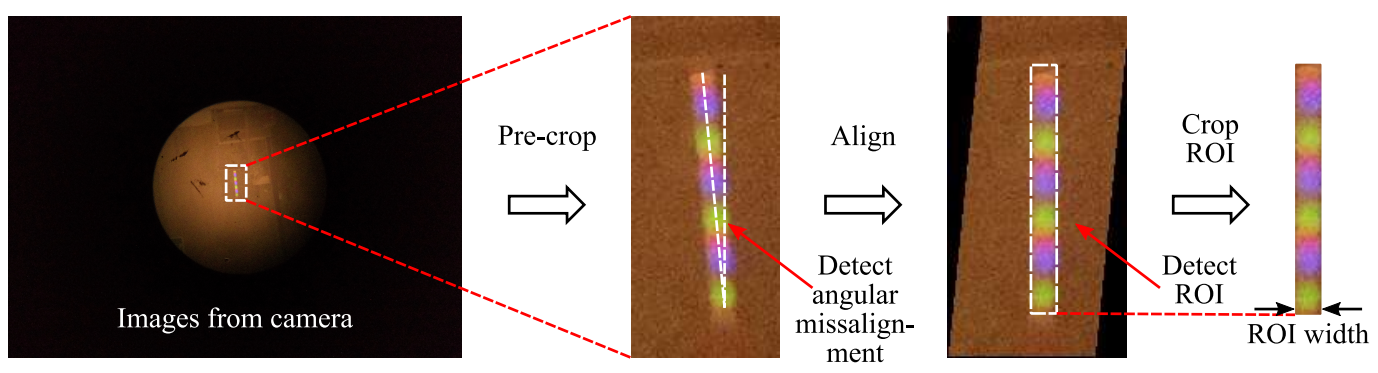

Fig. 3. Pre-processing stages of the images obtained with the $R_{x}$ module.

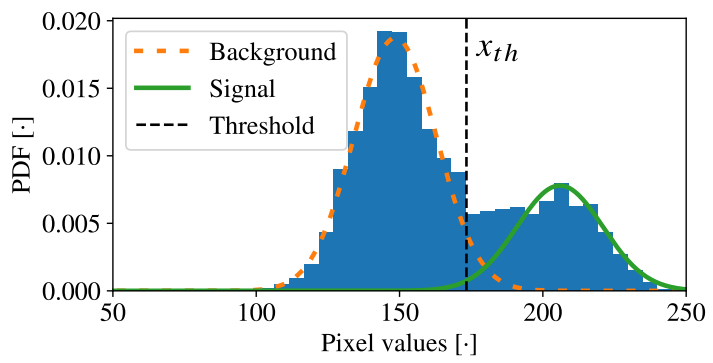

Fig. 4. Red channel histogram and fitted GMM curves of an arbitrary image frame captured at $d_{1}=100 \mathrm{~m}$ and $G_{V}=17.5 \mathrm{~dB}$.

with the SNR, is calculated as:

$$
r_{x y}=\frac{E[X T]-E[X] E[T]}{\sigma_{X} \sigma_{T}},
$$

where $X$ is the received signal or the detected ROI, and $T$ is a template of the expected waveform contained in the ROI, which is known because the $T_{x}$ module in this experiment is set to transmit a repetitive pattern. The coefficients $\sigma_{X}$ and $\sigma_{T}$ are the standard deviations of the signal and the template, respectively. Note that in this case, $\sigma_{T}$ is constant and greater than 0 , since the template is a series of RGB pulses. The operator $E[\cdot]$ refers to the expected value.

\section{RESULTS AND DISCUSSION}

In this section, the outcome of the processes explained in Section 3 are shown and reviewed, including the dust's impact on the ROI width, the SNR calculations, and the correlator outputs as measures of the signal quality.

In Fig. 5 it can be observed that the ROI is approximately $33 \%$ greater than $H_{0}$ in clear air, proving the assumption that multiple scattering significantly expands the PSF and the ROI consequently. The width expansion can be used for reducing the noise by taking the average value of the ROI pixels in the same row. These advantages are similar to the effect of camera defocusing employed by Eso et al. in [4]. According to the OOK modulation scheme employed, the image frames at $d_{1}$ contain up to 23 OOK symbols for each channel, while at $d_{2}$, they contain up to 14 symbols instead of 11 symbols of the theoretical clear air conditions. Due to the high resolution of the images and the image sensor's $t_{r s}$, a practical camera frame rate would be $15 \mathrm{fps}$, which is available in most commercial cameras. The achievable data rate of the system is then 1035 bps and $630 \mathrm{bps}$ at $100 \mathrm{~m}$ $200 \mathrm{~m}$, respectively. These values confirm the positive impact of the ROI expansion due to multiple scattering because, at long distances, the apparently-larger transmitter surface contains more symbols than in a scatter-free scenario (clear air).

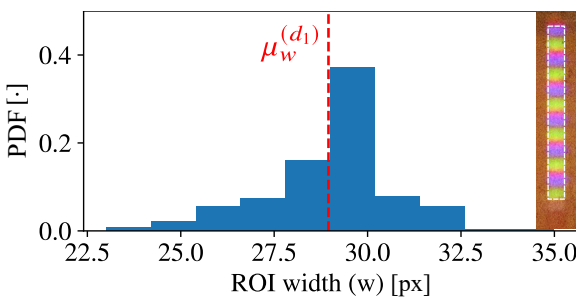

(a)

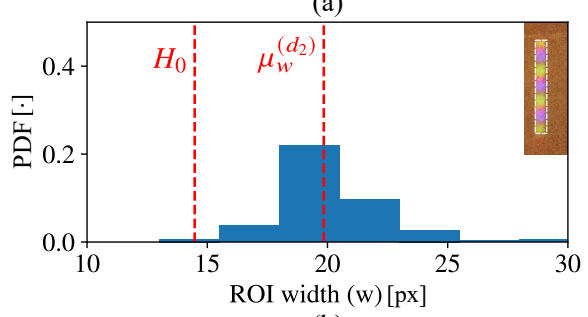

(b)

Fig. 5. Histograms showing the PDF of ROI width variation due to multiple scattering, where (a) corresponds to the results at distance $d_{1}=100 \mathrm{~m}$ and $(\mathrm{b}) d_{2}=200 \mathrm{~m}$. Insets in the graphs are examples of processed images, framing the detected ROI with a white dashed line.

Table 2. Extinction coefficient values under sandstorm measurements.

\begin{tabular}{lll}
\hline Channel & $\lambda[\mathrm{nm}]$ & $K_{\text {ext }}(\lambda)\left[\mathrm{m}^{-1}\right]$ \\
\hline Red & 630 & $7.2 \cdot 10^{-3}$ \\
\hline Green & 530 & $6.9 \cdot 10^{-3}$ \\
\hline Blue & 475 & $5.6 \cdot 10^{-3}$ \\
\hline
\end{tabular}

The image frames captured by the camera (at both $100 \mathrm{~m}$ and $200 \mathrm{~m}$, and using different values of gain allowed by the CMOS sensor) were processed to detect the OOK symbols, from which the SNR per each color channel was then calculated, as shown in the plots of Fig. 6. The channel extinction coefficients were computed from the symbol intensities at both distances using Eq. 2 and assuming $K_{\text {ext }}(\lambda)$ constant during the experiments. The resulting extinction coefficients under visibility $0.57 \mathrm{~km}$ are shown in Table 2. An SNR improvement of up to $\triangle S N R \approx$ $9 d B$ at $d_{1}$ and $3 \mathrm{~dB}$ at $d_{2}$ shows that the camera's analog gain positively impacts the SNR. The consequent BER values for each link span, according to Eq. 5 are then $9.14 \cdot 10^{-5}$ and $4.1 \cdot 10^{-3}$ for $100 \mathrm{~m}$ and $200 \mathrm{~m}$ respectively. The optimal gain in this high optical extinction scenario is the highest allowed by the camera of $20.6 \mathrm{~dB}$ and. The reason $G_{V}$ improves the SNR, in this case, is the high quantization noise due to the low received power, as explained by Eq. 6 . 


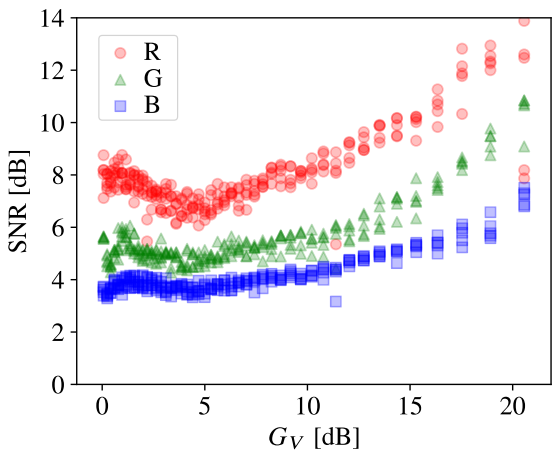

(a)

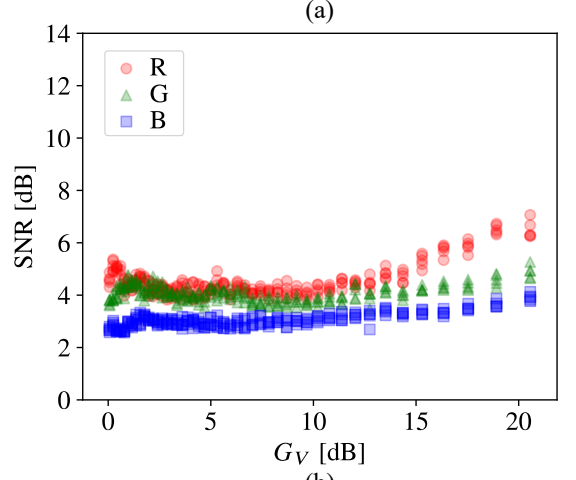

(b)

Fig. 6. Scatter plots of the R-G-B channels' SNR vs. the camera analog gain $\left(G_{V}\right)$, where (a) corresponds to the results at distance $d_{1}=100 \mathrm{~m}$ and (b) $d_{2}=200 \mathrm{~m}$.

The correlation values obtained by applying Eq. 11 to the image frames and the expected-waveform template are shown in Fig. 7. The correlation is observed to reach a plateau at high gains, which is always below 1.0 (perfect correlation). This occurs because SNR is finite, and Pearson's correlation coefficient can be statistically modeled as a ratio distribution between correlated numerator (first-order moment) and denominator (square root of a second-order moment). Furthermore, the resulting high correlation values occur because the template is large (44-pixel rows height), reducing the data variance.

The results presented show that the decoding stage can take advantage of the ROI expansion caused by the scattering and the improved image quality generated by the analog gain.

\section{CONCLUSIONS}

An OCC link using a commercial RS camera module receiver coupled to a telescope and a low power RGB LED transmitter was experimentally tested, during the sandstorm event that occurred on February 23rd, 2020, in the Canary Islands archipelago. During this experiment, the $\mathrm{AOD}_{550}$ reached values between 3.2 and 6.4, while the visibility dropped to approximately $0.57 \mathrm{~km}$. A comprehensive set of measurements was carried out, comprising image captures varying the camera analog gain at two target distances of $100 \mathrm{~m}$ and $200 \mathrm{~m}$. The link in these conditions can achieve data rates of $1035 \mathrm{bps}$ and $630 \mathrm{bps}$, respectively.

A channel model was derived for attenuated optical wireless signals in multiple scattering scenarios. The SNR computation for these types of links was used to assess sandstorms' effect on the evaluated OCC links. It is shown that SNR depends not only on shot noise but also on quantization noise, which can be mitigated by both the optical and camera's analog gain. This occurs because, for severely attenuated optical signals, the effective

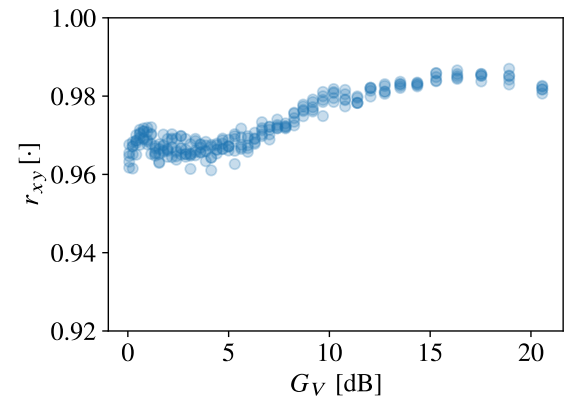

(a)

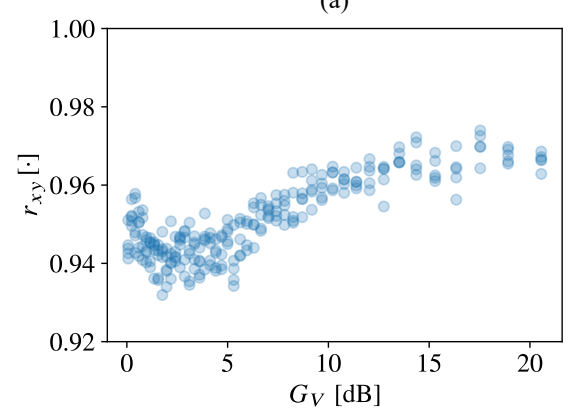

(b)

Fig. 7. Scatter plots for the correlation $\left(r_{x y}\right)$ vs. the camera analog gain $\left(G_{V}\right)$, where (a) corresponds to the results at distance $d_{1}=100 \mathrm{~m}$ and $(\mathrm{b}) d_{2}=200 \mathrm{~m}$.

number of bits in the analog-to-digital conversion are dramatically reduced. When electrically amplifying these signals, the extra noise due to the amplifier's noise figure is compensated by a higher ADC resolution. It was shown that the analog gain of the CMOS sensor improved the SNR under high attenuation conditions, approximately $9 \mathrm{~dB}$ in the $100 \mathrm{~m}$ experiment, and 3 $\mathrm{dB}$ in the $200 \mathrm{~m}$ experiment.

In order to accurately estimate SNR, a binary classification procedure based on a GMM was performed. This SNR calculation relies on the correct binary classification of ROI signal pixels versus the background. As it was experimentally demonstrated in [14], correlation-based metrics, on top of aiding the detection of ROI, can provide quick estimations about the signal quality. In this work, the same trends apply. It was observed that as the SNR increases, the values of $r_{x y}$ increase as well. The template-matching correlations obtained in this work are more than 0.92 because of its relatively-big dimensions, despite the optical distortions caused by the sandstorm.

It was observed that the GMM procedure, joint to a templatebased correlation, is suitable for detecting the ROI and estimating its dimensions. Taking advantage of this method, it was proved that sandstorms might generate ROI expansion due to multiple scattering. Concretely, during these experiments, the ROI was 33\% larger than expected. Considering that an RS-OCC system's maximum achievable data rate depends on the projected ROI size, this effect may be positive despite the increased path loss.

A highlighted finding of this work is that the scattering produced by the sandstorm particles allows expanding the effective area of the source projected in the image sensor. This provides room for allocating more symbols in the ROI than in a scatterfree scenario, thus increasing the achievable data rate. 


\section{FUNDING}

H2020 Marie Skłodowska-Curie Actions (764461).

\section{ACKNOWLEDGMENTS}

The authors thank the technical support received from Jaime Ticay and Cristo Jurado-Verdu from IDeTIC-ULPGC.

This project has received funding from the European Union's

Horizon 2020 research and innovation programme under the Marie Skłodowska-Curie grant agreement No 764461.

\section{DISCLOSURES}

The authors declare no conflicts of interest.

\section{REFERENCES}

1. IEEE Standard Association, "IEEE standard for local and metropolitan area networks-part 15.7: short-range wireless optical communication using visible light," 802.15.7-2011 (2011) pp. 1-309.

2. N. Saeed, S. Guo, K.-H. Park, T. Y. Al-Naffouri, and M.-S. Alouini, "Optical camera communications: Survey, use cases, challenges, and future trends," Phys. Commun. 37, 100900 (2019).

3. C. Jurado-Verdu, V. Matus, J. Rabadan, V. Guerra, and R. PerezJimenez, "Correlation-based receiver for optical camera communications," Opt. Express 27, 19150-19155 (2019).

4. E. Eso, S. Teli, N. B. Hassan, S. Vitek, Z. Ghassemlooy, and S. Zvanovec, "400 m rolling-shutter-based optical camera communications link," Opt. Lett. 45, 1059-1062 (2020).

5. V. Matus, V. Guerra, C. Jurado-Verdu, S. Teli, S. Zvanovec, J. Rabadan, and R. Perez-Jimenez, "Experimental evaluation of an analog gain optimization algorithm in optical camera communications," in 2020 12th International Symposium on Communication Systems, Networks Digital Signal Processing (CSNDSP), (2020), pp. 1-5.

6. E. Eso, A. Burton, N. Bani Hassan, M. M. Abadi, Z. Ghassemlooy, and S. Zvanovec, "Experimental Investigation of the Effects of Fog on Optical Camera-based VLC for a Vehicular Environment," in 2019 15th International Conference on Telecommunications (ConTEL), (IEEE, Graz, Austria, 2019), pp. 1-5.

7. N. Saha, M. S. Ifthekhar, N. T. Le, and Y. M. Jang, "Survey on optical camera communications: challenges and opportunities," IET Optoelectronics 9, 172-183(11) (2015).

8. P. Chavez-Burbano, V. Guerra, J. Rabadan, and R. Perez-Jimenez, "Optical camera communication for smart cities," in 2017 IEEE/CIC International Conference on Communications in China (ICCC Workshops), (2017), pp. 1-4.

9. M. Karbalayghareh, F. Miramirkhani, H. B. Eldeeb, R. C. Kizilirmak, S. M. Sait, and M. Uysal, "Channel modelling and performance limits of vehicular visible light communication systems," IEEE Transactions on Veh. Technol. 69, 6891-6901 (2020).

10. M. A. Khalighi and M. Uysal, "Survey on free space optical communication: A communication theory perspective," IEEE Commun. Surv. Tutorials 16, 2231-2258 (2014).

11. J. Horwath, D. D. Gonzalez, L. M. Navajas, A. L. Souto, V. Semerjyan, G. Raju, J. Grabowsky, C. Garcia, Y. Lai, T. Bähr, C.-C. Chen, F. Haque, A. Grier, H. Harding, M. Hunwardsen, E. Booen, and H. Hemmati, "Test results of error-free bidirectional 10 Gbps link for air-to-ground optical communications," in Free-Space Laser Communication and Atmospheric Propagation XXX, vol. $10524 \mathrm{H}$. Hemmati and D. M. Boroson, eds., International Society for Optics and Photonics (SPIE, 2018), pp. $417-430$.

12. J. Libich, J. Perez, S. Zvanovec, Z. Ghassemlooy, R. Nebuloni, and C. Capsoni, "Combined effect of turbulence and aerosol on free-space optical links," Appl. Opt. 56, 336-341 (2017).

13. Y. H. Kim, W. A. Cahyadi, and Y. H. Chung, "Experimental demonstration of vlc-based vehicle-to-vehicle communications under fog conditions," IEEE Photonics J. 7, 1-9 (2015).
14. V. Matus, E. Eso, S. R. Teli, R. Perez-Jimenez, and S. Zvanovec, "Experimentally derived feasibility of optical camera communications under turbulence and fog conditions," Sensors 20, 757 (2020).

15. T. Nguyen, A. Islam, and Y. M. Jang, "Region-of-interest signaling vehicular system using optical camera communications," IEEE Photonics J. 9, 1-20 (2017).

16. K. J. Ebrahim and A. Al-Omary, "Sandstorm effect on visible light communication," in 2017 9th IEEE-GCC Conference and Exhibition (GCCCE), (2017), pp. 1-7.

17. Z. Ghassemlooy, J. Perez, and E. Leitgeb, "On the performance of fso communications links under sandstorm conditions," in Proceedings of the 12th International Conference on Telecommunications, (2013), pp. 53-58.

18. H. Henniger and O. Wilfert, "An introduction to free-space optical communications." Radioengineering 19 (2010).

19. B. Cochenour, L. Mullen, and J. Muth, "Effect of scattering albedo on attenuation and polarization of light underwater," Opt. Lett. 35, 20882090 (2010).

20. D. Kedar and S. Arnon, "Urban optical wireless communication networks: The main challenges and possible solutions," Commun. Mag. IEEE 42, S2 - S7 (2004).

21. D. Kedar and S. Arnon, "The positive contribution of fog to the mitigation of pointing errors in optical wireless communication," Appl. Opt. 42, 4946-4954 (2003).

22. H. Le Minh, W. Popoola, and Z. Xu, "Techniques for enhancing the performance of VLC systems," Visible Light. Commun. Theory Appl. p. 195 (2017).

23. M. Carlowicz, "Dust blankets the canary islands," https://earthobservatory.nasa.gov/images/146337/dust-blanketsthe-canary-islands .

24. C. Pérez, K. Haustein, O. Jorba, Z. Janjic, N. Huneeus, J. Baldasano, T. Black, S. Basart, S. Nickovic, R. L. Miller, and et al., "Atmospheric dust modeling from meso to global scales with the online nmmb/bscdust model - part 1: model description, annual simulations and evaluation," Atmospheric chemistry physics 11, 13001-13027 (2011).

25. Sony Corporation, IMX219PQH5-C, Diagonal 4.60 mm (Type 1/4.0) 8 Mega-Pixel CMOS Image Sensor with Square Pixel for Color Cameras, Datasheet (Sony Corporation, 2014).

26. J. Shao and J. Mao, "Dust particle size distributions during spring in yinchuan, china," Adv. Meteorol. 2016 (2015). 